\title{
Identification and expression analysis on two forms bactericidal permeability- increasing protein (BPI)/lipopolysaccharide-binding protein (LBP) of Triangle mussel, Hyriopsis cumingii
}

\author{
Bao-qing $\mathrm{Hu}^{1}$, Chun-gen Wen ${ }^{1 *}$, Yi Liu ${ }^{2}$
}

1. School of Life Sciences, Nanchang University, Nanchang 330031, China

2. College of Life Sciences, Jiangxi Normal University, Nanchang 330022, Jiangxi, China

ABSTRACT Bactericidal permeability-increasing protein (BPI) and lipopolysaccharide-binding protein (LBP) are the numbers of the lipid transfer protein/lipopolysaccharide-binding protein family and play crucial roles in the innate immune response to Gram-negative bacteria. Two forms homolog of BPI/LBP, designed as Hc- BPI/LBP1 and Hc- BPI/LBP2, was cloned from the hemocyte cDNA of Hyriopsis cumingii. The complete cDNA of Hc- BPI/LBP1 included an open reading frame (ORF) of $1865 \mathrm{bp}$, and 3' and 5' untranslated regions (UTR's) of $139 \mathrm{bp}$ and $220 \mathrm{bp}$, respectively. The ORF encoded a putative protein of 479 amino acids with predicted 22-aa hydrophobic signal peptide. Hc- BPI/LBP2 cDNA was 2204 bp, 3' and 5' UTR's of $183 \mathrm{bp}$ and $464 \mathrm{bp}$, respectively. ORF encoded a polypeptide of 518 amino acids with a putative signal peptide of 22 amino acid residues. The deduced amino acid sequence contained an N-terminal BPI/LBP/CETP, link domain and a $\mathrm{C}$-terminal BPI/LBP/CETP domain BPI1 with three functional regions that display LPS-binding activity. The amino acids sequence similarity of LBP1 and LBP2 was 50\%. Hc- BPI/LBP1 and LBP2 transcripts could be detected in all normal tested tissues by quantitative real-time RT-PCR, including hepatopancreas, adductor muscle, mantle, gill and hemocytes, but the highest level of expression was in gills of HcBPI/LBP1 and in hepatopancreas of Hc- BPI/LBP2. The recombinant of HcBPI/LBPs showed a high affinity to LPS and can effectively kill Gram-negative bacteria.

\footnotetext{
${ }^{*}$ Corresponding author: Tel.: +86-0791-83969530; fax: +86-0791-83969530. E-mail address: cgwen@ncu.edu.cn (CG, Wen)
} 
Keywords: Bactericidal permeability-increasing protein, lipopolysaccharide-binding protein, Hyriopsis cumingii, Gene cloning, Expression 